\section{BMJ Open Respiratory Research}

\title{
Compliance after switching from CPAP to bilevel for patients with non- compliant OSA: big data analysis
}

Adam V Benjafield, ${ }^{1,2}$ Jean-Louis D Pepin, ${ }^{2,3,4}$ Kate Valentine, ${ }^{1,2}$ Peter A Cistulli, ${ }^{2,5}$ Holger Woehrle, ${ }^{2,6}$ Carlos M Nunez, ${ }^{1,2}$ Jeff Armitstead, ${ }^{2,7}$ Atul Malhotra ${ }^{2,8}$

\section{ABSTRACT}

To cite: Benjafield AV, Pepin J-LD, Valentine $\mathrm{K}$, et al. Compliance after switching from CPAP to bilevel for patients with non-compliant OSA: big data analysis. BMJ Open Resp Res 2019;6:e000380. doi:10.1136/ bmjresp-2018-000380

Received 11 November 2018 Revised 19 December 2018 Accepted 20 December 2018

Check for updates

(C) Author(s) (or their employer(s)) 2019. Re-use permitted under CC BY-NC. No commercial re-use. See rights and permissions. Published by BMJ.

${ }^{1}$ ResMed Science Center, San Diego, California, USA ${ }^{2}$ medXcloud Group, San Diego, California, USA ${ }^{3} \mathrm{HP} 2$ Laboratory, INSERM U1042, Université Grenoble Alpes, Grenoble, France ${ }^{4}$ EFCR Laboratory, Grenoble Alpes University Hospital, Grenoble, France

${ }^{5}$ Charles Perkins Centre, School of Medicine, University of Sydney and Royal North Shore Hospital, Sydney, New South Wales, Australia

${ }^{6}$ Sleep and Ventilation Center Blaubeuren, Lung Center UIm, Ulm, Germany

${ }^{7}$ ResMed Science Center, Sydney, New South Wales, Australia

${ }^{8}$ University of California San Diego, La Jolla, California, USA

Correspondence to Dr Atul Malhotra; amalhotra@ucsd.edu
Introduction For patients with obstructive sleep apnoea (OSA) who are initially non-compliant with continuous (automatic) positive airway pressure (CPAP/APAP) therapy, a bilevel PAP (Spont/VAuto) therapy transition pathway is available to improve therapy adherence. The aim of this retrospective study was to compare PAP therapy usage data of patients with non-compliant OSA (ncOSA) on CPAP/ APAP who were switched to bilevel PAP.

Methods A PAP telemonitoring database was queried between 1 January 2015 and 31 July 2016 for eligible patients started on CPAP/APAP and non-CMS (United States Center for Medicare and Medicaid Services) compliant and switched to bilevel PAP within 90 days of starting CPAP/APAP therapy. PAP therapy data on all patients were compared before switch (CPAP/APAP) and after switch (VAuto/Spont).

Results Of the 1496 patients with ncOSA identified, $30.3 \%$ used CPAP, $62.3 \%$ APAP, and $7.4 \%$ both APAP and CPAP before switching to a bilevel mode. $47.8 \%$ patients switched to Spont mode and $52.2 \%$ to VAuto mode. PAP usage significantly improved by $0.9 \mathrm{~h} /$ day $(\mathrm{p}<0.001)$ and all other device metrics (residual apnoea-hypopnoea index and unintentional mask leak) also improved after the switch. No patients had achieved US CMS criteria for compliance before the switch, and $56.8 \%$ did after. Conclusion This shows for the first time that there may be potential benefit from switching from CPAP/APAP to bilevel PAP for patients struggling with PAP adherence.

Nasal continuous positive airway pressure (CPAP) is a highly efficacious therapy for obstructive sleep apnoea (OSA). However, poor adherence to therapy can limit the effectiveness of treatment. ${ }^{1}$ Efforts to improve adherence to positive airway pressure (PAP) therapy are a key issue in OSA management. These include intensive support, ${ }^{2}$ changes in interface, nasal decongestion, cognitivebehavioural therapy and patient engagement techniques. ${ }^{3}$ Another option is to change the type of PAP mode, although existing data from observational studies and meta-analysis do not generally support the strategy of changing from one mode to another. ${ }^{4-8}$ However, current evidence is flawed by unselected populations and/or small numbers.

\section{Key messages}

What is the key question?

- Whether switching from continuous (automatic) positive airway pressure (CPAP/APAP) to bilevel PAP would be clinically beneficial in patients for whom initial therapy was deemed ineffective.

\section{What is the bottom line?}

> A switch to bilevel was associated with clinically and statistically significant improvements in PAP therapy adherence.

\section{Why read on?}

- There are few data showing the benefits of bilevel PAP over standard CPAP/APAP in patients with obstructive sleep apnoea who are pressure intolerant.

Our clinical experience suggests that patients who are pressure intolerant sometimes have a preference for use of either expiratory pressure relief or bilevel PAP modes in which a different pressure is provided during inspiration versus expiration. However, financial considerations can lead to questions regarding the value of switching patients to a more expensive device.

To our knowledge, there are few data showing the benefits of bilevel PAP over standard CPAP/automatic CPAP (APAP) in patients with OSA who are pressure intolerant. Therefore, we retrospectively investigated whether switching from CPAP/APAP to bilevel PAP would be clinically beneficial in patients for whom initial therapy was deemed ineffective. We used a big data approach in which records from individuals who had agreed to have their therapy device data stored using cloud-based technology were queried for potential changes in adherence.

We queried the AirView (ResMed) database for patients who were initiated on CPAP or APAP and switched to a bilevel PAP (VAuto or Spont mode) within the first 90 days after therapy initiation. The following 
device-derived parameters were determined before the switch (CPAP/APAP) and after the change to bilevel PAP: percentage of days with average daily PAP usage of $\geq 4$ hours; average daily PAP usage; residual apnoeahypopnoea index (as a measure of therapy effectiveness); leak; average PAP usage per session; percentage of patients achieving United States Center for Medicare and Medicaid Services (CMS) compliance criteria after switching to bilevel PAP therapy (CMS compliance is defined as device usage for $\geq 4$ hours/night on $70 \%$ of nights in a consecutive 30-day period). These criteria are important because they need to be met for a health insurance provider to continue paying for the treatment device. Essentially, if a patient does not meet CMS criteria for insurance coverage, they will lose their PAP device. Patients had not reached the definition of CMS compliance before the switch so that we could measure how many did then qualify after the switch.

Between 1 January 2015 and 31 July 2016, a total of 1496 eligible patients (mean age $58.1 \pm 14.2$ years) were identified from approximately 1.36 million patients; initial therapy was APAP in $62.3 \%$, CPAP in $30.3 \%$, and $\mathrm{APAP}$ and CPAP in $7.4 \%$. The pathway for CMS reimbursement of bilevel PAP after switching from APAP or CPAP is shown in figure 1. After switching, the bilevel PAP mode was VAuto in $52.2 \%$ of patients and Spont in $47.8 \%$. Average 95th percentile pressure before the switch (CPAP/APAP) was $12.5 \pm 3.7 \mathrm{cmH}_{2} \mathrm{O}$; after the switch to bilevel, average 95th percentile inspiratory and expiratory positive airway pressures were $15.1 \pm 4.1$ and $10.5 \pm 3.9 \mathrm{cmH}_{2} \mathrm{O}$, respectively. Switching to bilevel was associated with significant improvements in compliance and device metrics (table 1), regardless of bilevel PAP mode. Median average daily PAP usage improved by 0.9 $\mathrm{h} /$ day (figure 2). The greatest improvement was seen in those with the lowest average 95th percentile pressure (range 4-10 $\mathrm{cmH}_{2} \mathrm{O}$ ) with CPAP/APAP with an increase of $1.8 \mathrm{~h}$ /day, then the $10-15 \mathrm{cmH}_{2} \mathrm{O}$ range with $0.8 \mathrm{~h} /$ day increase followed by the $15-20 \mathrm{cmH}_{2} \mathrm{O}$ range with $0.7 \mathrm{~h} /$ day increase. Overall, $56.8 \%$ of patients who were non-compliant with CPAP/APAP achieved CMS compliance criteria after switching to bilevel PAP; the proportion of patients who achieved CMS criteria was 58.5\% in the subgroup of patients switched by day $60(59.9 \%$ of the total population) and $54.2 \%$ in the subgroup switched between day 61 and day $90(40.1 \%$ of the total population).

These findings are the first to show that there may be potential benefit from switching to bilevel therapy from CPAP or APAP for patients who are struggling with PAP adherence. To the best of our knowledge, most prior studies in this area were small and based on determination of non-inferiority rather than superiority. The majority of existing literature has suggested that bilevel therapy is equivalent to CPAP. We know of only one small study that showed potential superiority with bilevel PAP therapy. ${ }^{9}$ However, it focused on a proprietary technology and the study was small and showed only modest benefits.

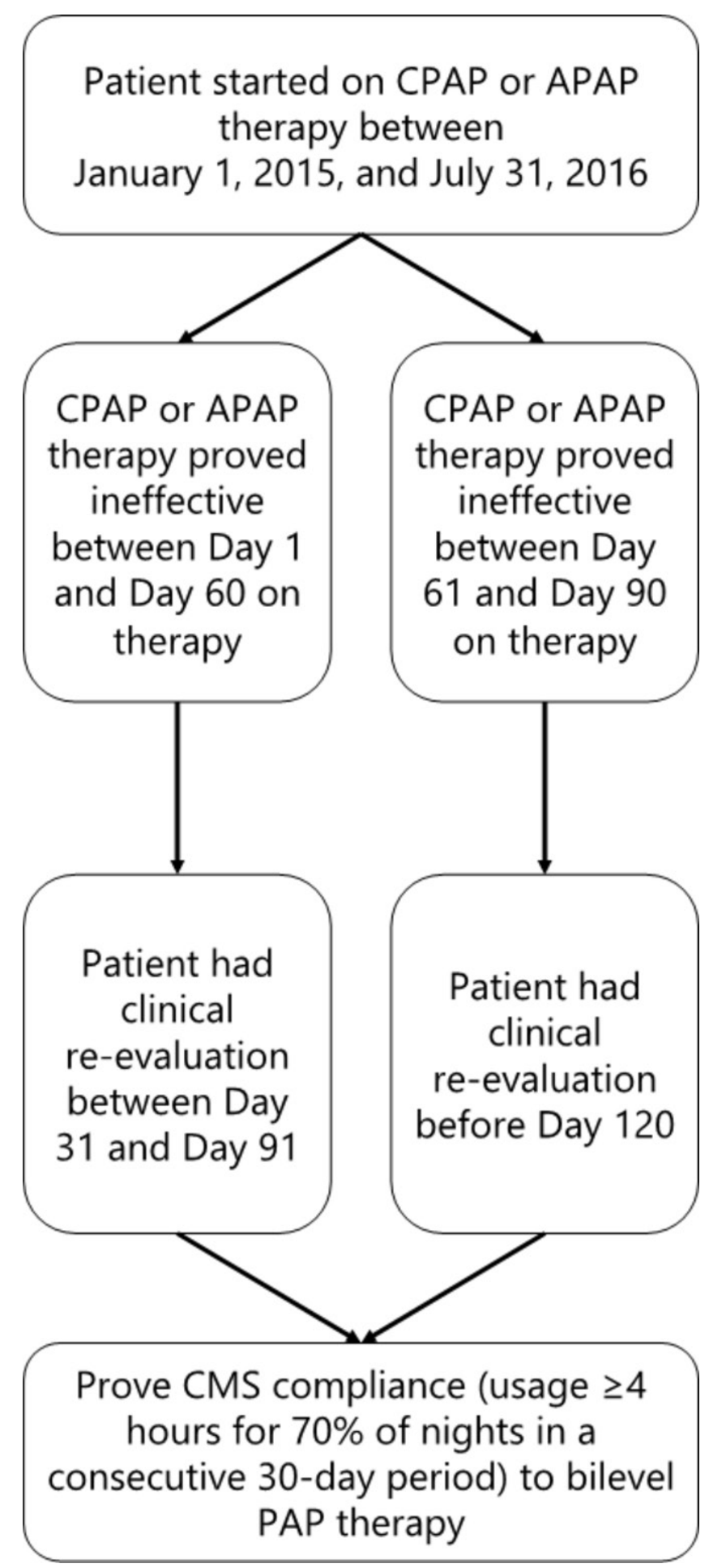

Figure 1 US Center for Medicare and Medicaid Services (CMS) bilevel reimbursement pathway. APAP, automatic continuous positive airway pressure; CPAP, continuous positive airway pressure; PAP, positive airway pressure.

In our study, a switch to bilevel was associated with clinically and statistically significant improvements in PAP therapy adherence with greatest improvements seen in those on lower pressures and those switched before day 60 . The study was specifically designed around the US CMS PAP adherence criteria, which require ongoing use 
Table 1 Changes in compliance and device metrics

\begin{tabular}{lccrc}
\hline & Before switch (CPAP/APAP) & After switch (bilevel PAP) & Difference & P value \\
\hline Average AHI & $4.9(2.0,11.8)$ & $4.0(1.7,9.2)$ & -0.9 & $<0.001$ \\
Average median leak, L/min & $5.0(1.3,11.3)$ & $4.1(1.1,10.4)$ & -0.9 & $<0.001$ \\
\% Days with usage of $\geq 4$ hours & $52.7(14.3,86.9)$ & $68.9(27.8,93.3)$ & +16.2 & $<0.001$ \\
\hline
\end{tabular}

Values are median (IQR).

AHI, apnoea-hypopnoea index; APAP, automatic continuous positive airway pressure; CPAP, continuous positive airway pressure; PAP, positive airway pressure.

of PAP therapy for patients to keep the equipment. The goal was to identify strategies that could impact clinical care under current reimbursement pathways. As such, the observed improvement in adherence to therapy associated with a switch to bilevel PAP could allow a substantial proportion of patients (up to $57 \%$ in this study) who would otherwise not have met CMS criteria to keep their funded PAP device rather than have to discontinue or pay for therapy. Thus, there are clear benefits to patients who improve adherence in this context. Achieving CMS criteria means that patients are able to retain their funded PAP therapy and continue to benefit from treatment. If CMS criteria are not met and the patient does not choose to pay for therapy, they would have to go through the complete clinical pathway of physician consultation, sleep study, diagnosis and therapy initiation if they wanted to try again to access funded CPAP therapy.

Although our study had a number of strengths, we also acknowledge some limitations. It was not a randomised trial and therefore we cannot rule out the possibility that patients who switched to bilevel PAP were systematically different from those who did not. In addition, we cannot exclude the possibility that other adherence-improving measures were implemented at the time of the switch to bilevel PAP nor do we know the mechanisms of the benefit. In theory, frequent visits to a healthcare provider could have intangible benefits to patients that may be as important as the change in PAP technology. Given that patients were selected based on poor compliance, regression to the mean could also contribute to some of the improvements in compliance seen in our study. However, early device usage

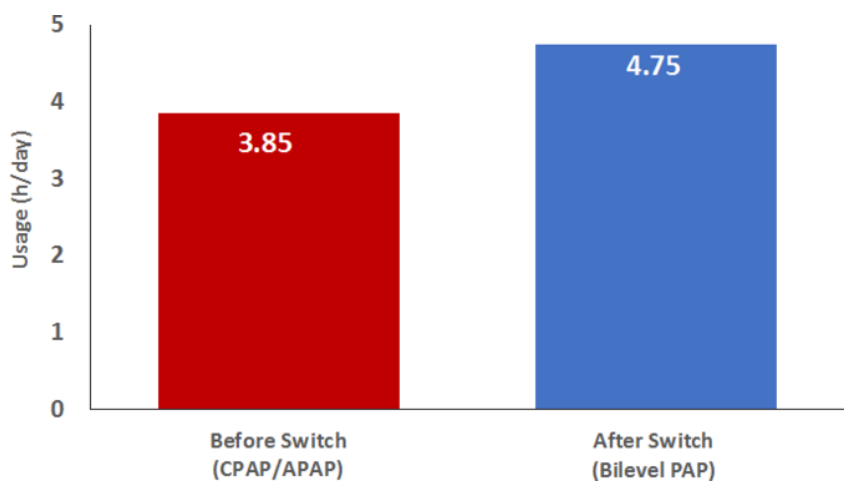

Figure 2 Median average daily positive airway pressure device usage. APAP, automatic continuous positive airway pressure; CPAP, continuous positive airway pressure; PAP, positive airway pressure. tends to predict longer-term usage, so the contribution of regression to the mean to the increase in device usage seen in our analysis may be small. Based on our study design and the nature of the de-identified database queried, we do not know specific details about our participants and cannot determine whether the observed benefits vary by factors such as age, race, gender or socioeconomic status. Thus, further research is also recommended in this area. Finally, we did not have the ability to assess major outcomes and therefore do not know whether the change to bilevel therapy had any beneficial effects on OSA-related symptoms or comorbidities. However, improvements in PAP device usage of at least $0.5 \mathrm{~h}$ /day have been defined as being clinically relevant. ${ }^{9}$ Therefore, the $0.9 \mathrm{~h}$ /day improvement in device usage after a switch to bilevel PAP therapy in our study is considered likely to be associated with some benefits for patients.

Acknowledgements The authors wish to thank the following for statistical analysis: Yang Yan M.Sc.Stat and Jingjing Li M.Sc.Stat. Manuscript formatting assistance was provided by Nicola Ryan, independent medical writer, funded by ResMed.

Contributors AVB contributed to the conception and design of the study, acquisition and interpretation of the data, and to the manuscript revision. J-LDP, PAC, HW, CMN and JA contributed to data interpretation and to the manuscript revision. KV contributed to the acquisition and interpretation of the data and to the manuscript revision. AM contributed to data interpretation and to drafting the manuscript. All authors reviewed and commented on the manuscript.

Funding This study was funded by ResMed Corp.

Competing interests AVB, KV, CMN and JA are all employees of ResMed. $J$-LDP is supported by the French National Research Agency in the framework of the 'Investissements d'avenir' program (ANR-15-IDEX-02). His department has received research support from Philips Respironics, Fisher and Paykel, and ResMed. PAC has an appointment to an endowed academic Chair at the University of Sydney that was established from ResMed funding. He has received research support from ResMed, SomnoMed and Zephyr Sleep Technologies. He is a consultant/adviser to Zephyr Sleep Technologies, and Narval. He has a pecuniary interest in SomnoMed related to a previous role in R\&D (2004). HW has received consulting/speaking fees from ResMed and Inspire Medical. AM relinquished all outside personal income as an Officer of the ATS in 2012. ResMed gave a philanthropic donation to UC San Diego, but AM receives no personal income from ResMed.

Patient consent for publication Not required.

Ethics approval The Chesapeake Institutional Review Board (IRB) determined that this research was exempt from IRB oversight because the data used were de-identified and participants had agreed to having their data stored on a cloudbased server.

Provenance and peer review Not commissioned; externally peer reviewed Data sharing statement No additional data are available.

Open access This is an open access article distributed in accordance with the Creative Commons Attribution Non Commercial (CC BY-NC 4.0) license, which permits others to distribute, remix, adapt, build upon this work non-commercially, and license their derivative works on different terms, provided the original work is properly cited, appropriate credit is given, any changes made 
indicated, and the use is non-commercial. See: http://creativecommons.org/ licenses/by-nc/4.0/.

\section{REFERENCES}

1. Weaver TE, Mancini C, Maislin G. Continuous positive airway pressure treatment of sleepy patients with milder obstructive sleep apnea: results of the CPAP apnea trial North American program (CATNAP) randomized clinical trial. Am J Respir Crit Care Med 2012;86:677-83.

2. Hoy CJ, Vennelle M, Kingshott RN, et al. Can intensive support improve continuous positive airway pressure use in patients with the sleep apnea/hypopnea syndrome? Am J Respir Crit Care Med 1999;159:1096-100.

3. Malhotra A, Crocker ME, Willes L, et al. Patient engagement using new technology to improve adherence to positive airway pressure therapy: a retrospective analysis. Chest 2018;153:843-50.

4. Ballard RD, Gay PC, Strollo PJ. Interventions to improve compliance in sleep apnea patients previously non-compliant with continuous positive airway pressure. J Clin Sleep Med 2007;3:706-12.
5. Carlucci A, Ceriana P, Mancini M, et al. Efficacy of bilevel-auto treatment in patients with obstructive sleep apnea not responsive to or intolerant of continuous positive airway pressure ventilation. $J$ Clin Sleep Med 2015;11:981-5.

6. Gay PC, Herold DL, Olson EJ. A randomized, double-blind clinical trial comparing continuous positive airway pressure with a novel bilevel pressure system for treatment of obstructive sleep apnea syndrome. Sleep 2003;26:864-9.

7. Powell ED, Gay PC, Ojile JM, et al. A pilot study assessing adherence to auto-bilevel following a poor initial encounter with CPAP. J Clin Sleep Med 2012;8:43-7.

8. Smith I, Lasserson TJ. Pressure modification for improving usage of continuous positive airway pressure machines in adults with obstructive sleep apnoea. Cochrane Database Syst Rev 2009:4:CD003531.

9. Kapur VK, Auckley DH, Chowdhuri S, et al. Clinical practice guideline for diagnostic testing for adult obstructive sleep apnea: an American Academy of Sleep Medicine clinical practice guideline. J Clin Sleep Med 2017;13:479-504. 